\title{
¿Implicar a los estudiantes en la modificación de rúbricas influye sobre su percepción de validez?
}

\begin{abstract}
Jose Ignacio Priego-Quesada ${ }^{\text {a,b }}$, Lara Requena-Bueno ${ }^{\text {a }}$, Irene Jimenez-Perez ${ }^{\text {a,b }}$ y Rafael García-Ros $^{\mathrm{c}}$

${ }^{a}$ Grupo de Investigación en Biomecánica aplicada al Deporte (GIBD), Departamento de Educación Física y Deportiva, Facultad de Ciencias de la Actividad Física y el Deporte, Universitat de València, ${ }^{\mathrm{b}}$ Grupo de Investigación en Física Médica (GIFIME), Departamento de Fisiología, Facultad de Medicina y Odontología, Universitat de València y 'IQDOCENT Grupo de Innovación Educativa, Departamento de Psicología Evolutiva y de la Educación, Facultad de Psicología, Universitat de València. Emails: j.ignacio.priego@uv.es; 1.requena.gibd@gmail.com; i.jimenez.gibd@gmail.com; $\underline{\text { Rafael.Garcia@uv.es }}$
\end{abstract}

Abstract: Oral presentation is one of the fundamental competences in the teaching-learning process for most of the university students and rubrics could be used as a useful tool in this process. Therefore, the main objective was to determine the perception of validity and utility of a rubric for students of the Physical Education and Sports Sciences degree, considering also if they were involved or not in the rubric's development. 115 students participated, in two groups. The teacher of the subject gave to both groups the same rubric to evaluate their oral presentations. So, one of the groups modified and prepared the rubric's elements. After oral presentations, both groups completed a rubric's validity perception questionnaire. Moreover, the academic performance of the two groups was recorded. Students positively assessed the rubric's validity as a method of preparing and evaluating the oral presentation. The results non-show to the possible effect on this perception in the students that could be involved in the modification and preparation of the rubric. However, academic performance could have a greater influence.

Keywords: rubrics, higher education, validity perception, learning, evaluation, oral presentation. 


\begin{abstract}
Resumen
Una de las competencias transversales fundamentales en el ámbito universitario es la exposición oral. Las rúbricas pueden ser una herramienta especialmente útil para promover su desarrollo y alinear los procesos enseñanza-aprendizaje y la evaluación de la misma. El objetivo principal del estudio ha consistido en determinar la validez y utilidad que los estudiantes de la titulación de Ciencias de la Actividad Física y el Deporte conceden a una rúbrica desarrollada al efecto, considerando adicionalmente su implicación o no en la elaboración de la misma. En el estudio participaron un total de 115 estudiantes divididos en dos grupos. El profesor de la asignatura facilitó la misma rúbrica a ambos grupos para guiar el desarrollo y evaluar sus posteriores exposiciones orales, aunque solo un grupo pudo participar en la elaboración y modificación de los elementos que componen la rúbrica. Una vez realizadas las exposiciones orales, ambos grupos respondieron a un cuestionario de percepción de validez de la rúbrica. Además, se tuvo constancia del rendimiento académico de los dos grupos. Ambos grupos valoraron positivamente la validez y utilidad de la rúbrica como método de preparación y evaluación de la exposición oral. Sin embargo, los resultados no muestran efectos significativos de la implicación o no en la modificación y elaboración de la rúbrica sobre la misma, a diferencia del rendimiento académico que sí que pudo tener una mayor influencia.
\end{abstract}

Palabras clave: rúbricas, enseñanza universitaria, percepción validez, aprendizaje, evaluación, exposición oral. 


\section{Introducción}

Tradicionalmente, la evaluación ha sido considerada en la enseñanza universitaria como una fase instruccional exclusivamente dirigida a constatar el nivel de conocimientos alcanzado por los alumnos, habitualmente desgajada de los procesos de enseñanza-aprendizaje de los mismos. Sin embargo, coincidiendo con la perspectiva del Espacio Europeo de Educación Superior, en los últimos años se ha efectuado un especial énfasis en la consideración de métodos de enseñanza activos, centrados en el aprendiz, donde la evaluación también constituye una herramienta instruccional especialmente valiosa para potenciar los procesos de enseñanza-aprendizaje y el desarrollo de las competencias implicadas en las distintas materias y titulaciones (García-Ros 2011). Desde esta perspectiva general, la exposición oral constituye una de las competencias transversales fundamentales que deben adquirir los estudiantes universitarios (Crosling y Ward 2002, Dunbar et al. 2006, Živković 2014). Sin embargo, tanto enseñarla como aprenderla es una tarea compleja (Dasí e Iborra 2007) y las rúbricas constituyen una herramienta de evaluación fundamental para alinear los procesos de enseñanza, aprendizaje y evaluación de la misma (Dunbar et al. 2006).

Las rúbricas pueden definirse como una guía que articula de forma clara los criterios utilizados para evaluar la calidad y el nivel de desarrollo competencial alcanzado por los estudiantes (Reddy y Andrade 2010). De forma general, las rúbricas se conforman por tres elementos esenciales: criterios de evaluación, descriptores y/o niveles de calidad y estrategias de calificación o puntuación (Reddy y Andrade 2010). Algunos autores destacan que entre los beneficios de utilizar rúbricas pueden destacarse: a) una mayor calidad en el aprendizaje, dada la focalización de la atención sobre los criterios positivos en la elaboración de los trabajos, b) una autoevaluación de los estudiantes más sencilla y precisa, c) las evaluaciones sobre el nivel de adquisición de las competencias por parte de los docentes son más válidas $\mathrm{y}, \mathrm{d})$ poder ofrecer un feedback más específico al alumnado sobre la calidad de sus elaboraciones o ejecuciones, destacando los aspectos a mejorar (Jonsson y Svingby 2007, García-Ros 2011). Por otro lado, se ha observado que la evaluación mediante rúbricas reduce el número de reclamaciones por parte del alumnado sobre su calificación (Barney et al. 2012).

La utilización de las rúbricas ha sido valorada positivamente por parte del alumnado en diferentes estudios (Andrade y Du 2005, Barney et al. 2012, Eshun y Osei-Poku 2013). De manera específica, Andrade y Du (2005) observaron que los estudiantes valoraban de manera positiva las rúbricas como herramienta para obtener mejores calificaciones, reducir la ansiedad y mejorar la calidad del trabajo. Sin embargo, para muchos no se trató tanto de mejorar su competencia, sino de conseguir realizar lo que el profesorado quería (Andrade y Du 2005). En este sentido, es recomendable conocer la valoración específica del alumnado sobre la validez y utilidad de las rúbricas que se aplican en una intervención educativa. Por último, aunque se sugiere que implicar al alumnado en la elaboración de rúbricas tiene un efecto positivo sobre la autorregulación y autoeficacia hacia el aprendizaje y ejecución académica (Fraile, Panadero y Pardo, 2017), resultan escasos los trabajos que analizan los efectos de esta implicación y cómo puede afectar esta cuestión a su percepción de la validez y utilidad de las rúbricas. 


\section{Objetivos}

El estudio pretendió determinar la percepción de validez que conceden los estudiantes universitarios de Ciencias de la Actividad Física y del Deporte a una rúbrica dirigida a evaluar la calidad de sus exposiciones orales, identificando adicionalmente las posibles diferencias en su percepción validez en función de su implicación o no en la modificación de la misma.

Debido a esto, el objetivo del presente trabajo fue conocer si existían diferencias en la percepción de validez de una rúbrica para la evaluación de la exposición oral, entre un grupo que había sido implicado en la elaboración de la rúbrica y otro al que se le había asignado la rúbrica sin posibilidad de ser modificada.

\section{Desarrollo de la innovación}

\subsection{Alumnado sobre el que se ha desarrollado la intervención}

La intervención se llevó a cabo en el curso 2018-2019, en la asignatura "Metodología de la Enseñanza de la Actividad Física y el Deporte" del Grado de Ciencias de la Actividad Física y el Deporte, de la Universitat de València. Dicha asignatura se imparte en el primer cuatrimestre del tercer curso del grado. El alumnado se divide en dos grupos según el idioma de impartición de la materia, castellano y valenciano, teniendo cada uno de éstos 45 y 70 estudiantes matriculados, respectivamente, en el presente curso analizado.

\subsection{Diseño de la intervención}

La intervención se puso en práctica en el tema de la asignatura donde se desarrollan los diferentes estilos de enseñanza aplicables a las ciencias de la actividad física y el deporte. En este tema, el profesor imparte tres clases y deja el resto de sesiones para que los alumnos presenten los diferentes estilos de enseñanza mediante exposiciones orales. En la primera clase, el profesor realizó una introducción del tema. En la segunda entrenó al alumnado en el uso de la rúbrica y le hizo partícipe en su elaboración (únicamente en el caso de uno de los grupos, como se detallará más adelante). Y en la tercera proporcionó conclusiones para cerrar el tema. Entre la segunda y tercera clase, se llevaron a cabo las exposiciones orales por parte del alumnado. Los grupos de castellano y valenciano realizaron un total de 8 y 9 exposiciones orales, respectivamente. La normativa de las exposiciones orales consistió en los siguientes puntos:

- La composición de los equipos la realizó el profesor siguiendo un criterio de orden alfabético en la lista.

- Los equipos tenían un número de componentes de entre 5 y 7.

- La duración de la exposición debía ser entre 25 y 35 minutos.

- El mismo día, justo antes de la exposición, el equipo debía dar un folio al profesor con la división de la exposición según el número de componentes, y el profesor decidir qué miembro debía exponer cada una de las partes. El objetivo era que todos los componentes tuviesen que conocer la presentación al completo. 
- En cada presentación oral, tanto el profesor como el alumnado que no realizaban la exposición, debían evaluar al equipo mediante la utilización de una rúbrica (sección 3.3). La nota final de la exposición oral fue del $60 \%$ por parte del profesor, del $30 \%$ por parte del alumnado, y un $10 \%$ en forma de autoevaluación.

- Antes de que el alumnado conociese su calificación obtenida, se les pidió que cumplimentaran un cuestionario anónimo sobre la percepción de validez de la rúbrica (sección 3.4).

En la sesión en la que se realizó el entrenamiento sobre el uso de la rúbrica, se llevó a cabo una intervención diferente con cada grupo; por lo que el grupo de castellano a partir de ahora se llamará INTERVENCIÓN, y el grupo de valenciano CONTROL. En primer lugar, a ambos grupos se les proporcionó la rúbrica y visualizaron un vídeo de una exposición oral que tuvieron que valorar mediante el empleo de la rúbrica. Tras esa primera valoración, ambos grupos pudieron preguntar las dudas que tuviesen sobre los criterios de evaluación considerados en la rúbrica, así como sobre los niveles de ejecución considerados en los mismos y la estrategia de calificación utilizada. Sin embargo, al grupo INTERVENCIÓN se le dio adicionalmente la opción de modificar los distintos componentes destacados (criterios, niveles de ejecución y estrategia de calificación). Así, en cada ítem se fue debatiendo si modificar o no alguno de sus aspectos, introduciendo en su caso las modificaciones pertinentes destacadas por los estudiantes. Tras este proceso, ambos grupos visualizaron un segundo vídeo y lo volvieron a evaluar utilizando la rúbrica, en el caso del grupo INTERVENCIÓN utilizando ya la rúbrica modificada por ellos.

La distribución temporal de la intervención se muestra en la Tabla 1.

Tabla 1. Distribución temporal de la intervención.

\begin{tabular}{|c|c|}
\hline Momento & Fecha \\
\hline $\begin{array}{l}\text { Entrenamiento rúbrica de ambos grupos y } \\
\text { participación en su elaboración por parte del } \\
\text { grupo INTERVENCIÓN }\end{array}$ & $25 / 09 / 2018$ \\
\hline Realización exposiciones orales & Del 04/10/2018 al 23/10/2018 \\
\hline $\begin{array}{c}\text { Realización cuestionario percepción validez } \\
\text { rúbrica }\end{array}$ & $25 / 10 / 2018$ \\
\hline Publicación notas exposición oral & $5 / 11 / 2018$ \\
\hline
\end{tabular}

\subsection{Rúbrica}

Se utilizó como rúbrica para valorar la exposición oral en el grupo CONTROL, la desarrollada y evaluada por García-Ros (2011) (Tabla 2). El grupo INTERVENCIÓN modificó aspectos de la rúbrica relacionados con el apartado de apoyo visual (Tabla 3). Aunque se analizó ítem por ítem con el grupo INTERVENCIÓN y se le incentivó todo lo posible a que realizasen cambios, en la mayoría de los ítems valoraron que eran adecuados y no era necesario modificarlos. 
Tabla 2. Rúbrica utilizada (García-Ros 2011) para las exposiciones orales del grupo CONTROL.

\begin{tabular}{|c|c|c|c|c|}
\hline \multicolumn{5}{|c|}{ 1. Principios básicos $(30 \%)$} \\
\hline & Inadecuado 0 & A mejorar 1 & Adecuado 2 & Muy adecuado 3 \\
\hline $\begin{array}{l}\text { Dominio y } \\
\text { comprensión del } \\
\text { tema }\end{array}$ & $\begin{array}{l}\text { No domina ni comprende el } \\
\text { tema }\end{array}$ & $\begin{array}{c}\text { Muestra una buena } \\
\text { comprensión de partes del } \\
\text { tema, pero no de alguna de } \\
\text { ellas }\end{array}$ & $\begin{array}{l}\text { Muestra una buena comprensión } \\
\text { del tema }\end{array}$ & $\begin{array}{l}\text { Manifiesta una comprensión } \\
\text { completa y profunda del tema }\end{array}$ \\
\hline $\begin{array}{l}\text { Planificación y } \\
\text { organización del } \\
\text { contenido }\end{array}$ & No ha planificado & $\begin{array}{c}\text { Discurso dificil de seguir y } \\
\text { conexiones incoherentes, lo } \\
\text { que indica planificación pobre } \\
\text { y que ha dedicado poco } \\
\text { esfuerzo }\end{array}$ & $\begin{array}{c}\text { Sigue un esquema general } \\
\text { adecuado, aunque alguna vez se } \\
\text { pierde o necesita prestar más } \\
\text { atención a detalles }\end{array}$ & $\begin{array}{c}\text { Discurso bien planificado, } \\
\text { enlaza los tópicos de manera } \\
\text { lógica y coherente }\end{array}$ \\
\hline $\begin{array}{l}\text { Integración } \\
\text { elementos } \\
\text { importantes }\end{array}$ & Inadecuada integración & $\begin{array}{c}\text { Escasa integración de los } \\
\text { elementos fundamentales de la } \\
\text { temática }\end{array}$ & $\begin{array}{l}\text { Incorpora de forma eficaz los } \\
\text { elementos importantes del tema } \\
\text { la mayor parte de la presentación }\end{array}$ & $\begin{array}{l}\text { Incorpora de forma eficaz los } \\
\text { elementos importantes del } \\
\text { tema en toda la presentación }\end{array}$ \\
\hline \multicolumn{5}{|c|}{ 2. Aplicación y ejemplificación $(30 \%)$} \\
\hline & Inadecuado 0 & A mejorar 1 & Adecuado 2 & Muy adecuado 3 \\
\hline $\begin{array}{l}\text { Coherencia con } \\
\text { el modelo } \\
\text { teórico }\end{array}$ & $\begin{array}{l}\text { E1 ejemplo desarrollado no es } \\
\text { coherente con el modelo }\end{array}$ & $\begin{array}{l}\text { El ejemplo desarrollado es } \\
\text { coherente con el modelo, } \\
\text { aunque diversos aspectos no } \\
\text { quedan recogidos } \\
\text { adecuadamente }\end{array}$ & $\begin{array}{c}\text { El ejemplo desarrollado es } \\
\text { coherente con el modelo teórico, } \\
\text { aunque necesitaria afinar algún } \\
\text { aspecto concreto }\end{array}$ & $\begin{array}{l}\text { E1 ejemplo desarrollado es } \\
\text { coherente con el modelo } \\
\text { teórico }\end{array}$ \\
\hline $\begin{array}{l}\text { Clarificación } \\
\text { modelo y } \\
\text { motivación } \\
\text { audiencia }\end{array}$ & $\begin{array}{l}\text { El ejemplo seleccionado es } \\
\text { confuso y no se ajusta al } \\
\text { modelo }\end{array}$ & $\begin{array}{l}\text { El ejemplo no clarifica } \\
\text { adecuadamente y no resulta } \\
\text { motivante para la audiencia }\end{array}$ & $\begin{array}{l}\text { E1 ejemplo no clarifica } \\
\text { adecuadamente o resulta } \\
\text { motivante para la audiencia }\end{array}$ & $\begin{array}{l}\text { E1 ejemplo resulta clarificador } \\
\text { y motivante para la audiencia }\end{array}$ \\
\hline
\end{tabular}




\begin{tabular}{|c|c|c|c|c|}
\hline \multicolumn{5}{|c|}{ 3. Apoyo visual $(20 \%)$} \\
\hline & Inadecuado 0 & A mejorar 1 & Adecuado 2 & Muy adecuado 3 \\
\hline $\begin{array}{c}\text { Número y } \\
\text { explicación de } \\
\text { las diapositivas }\end{array}$ & $\begin{array}{l}\text { No cumple requisitos de } \\
\text { número y adecuación en su } \\
\text { explicación. }\end{array}$ & $\begin{array}{l}\text { Utiliza un número inadecuado } \\
\text { de diapositivas, aunque intenta } \\
\text { explicarlas }\end{array}$ & $\begin{array}{c}\text { Cantidad de diapositivas } \\
\text { adecuada, aunque imprecisa } \\
\text { explicación de alguna de ellas }\end{array}$ & $\begin{array}{l}\text { Cantidad de diapositivas } \\
\text { adecuada y explicación } \\
\text { pertinente de las mismas }\end{array}$ \\
\hline $\begin{array}{l}\text { Adecuación de } \\
\text { las diapositivas }\end{array}$ & $\begin{array}{l}\text { Incorpora diapositivas no } \\
\text { pertinentes con la temática, no } \\
\text { facilitan la comprensión ni la } \\
\text { motivación de la audiencia. }\end{array}$ & $\begin{array}{c}\text { Aunque la mayoría de ellas } \\
\text { están relacionadas con el tema, } \\
\text { no favorecen su comprensión } \\
\text { ni la motivación de la } \\
\text { audiencia }\end{array}$ & $\begin{array}{l}\text { Todas tiene relación con el tema } \\
\text { y facilitan que la comprensión } \\
\text { material, aunque algunas no } \\
\text { están conectadas con el discurso } \\
\text { o adolecen de amenidad }\end{array}$ & $\begin{array}{l}\text { Todas tiene relación con el } \\
\text { tema y conexión con el } \\
\text { discurso, facilitando la } \\
\text { comprensión del material y } \\
\text { resultan amenas/captan la } \\
\text { atención }\end{array}$ \\
\hline Legibilidad & $\begin{array}{c}\text { Ilegible, uso de colores } \\
\text { abrumador, abusa de texto y } \\
\text { de párrafos }\end{array}$ & $\begin{array}{c}\text { Letra legible por el } 85 \% \\
\text { audiencia aproximad., } \\
\text { extensión excesiva en gran } \\
\text { parte diapositivas. Inadecuado } \\
\text { contraste de colores }\end{array}$ & $\begin{array}{c}\text { Letra legible para toda la } \\
\text { audiencia, más de } 5 \text { lineas por } \\
\text { diapositiva. Contraste adecuado } \\
\text { de colores }\end{array}$ & $\begin{array}{l}\text { No más de } 5 \text { palabras por } \\
\text { línea, ni más de } 5 \text { lineas por } \\
\text { diapositiva, letra legible para } \\
\text { toda la audiencia, contraste } \\
\text { adecuado de colores }\end{array}$ \\
\hline $\begin{array}{c}\text { Relevancia y } \\
\text { adecuación } \\
\text { imágenes/esque } \\
\text { mas } \\
\end{array}$ & No utiliza o distraen & $\begin{array}{c}\text { Acorde con contenido, aunque } \\
\text { no relevantes y de poca } \\
\text { resolución }\end{array}$ & $\begin{array}{c}\text { Relevantes, acordes con } \\
\text { contenidos en la } \\
\text { mayoría de ocasiones, pero poca } \\
\text { resolución } \\
\end{array}$ & $\begin{array}{c}\text { Relevantes, acordes con } \\
\text { contenidos y con adecuada } \\
\text { resolución }\end{array}$ \\
\hline $\begin{array}{l}\text { Secuenciación, } \\
\text { animaciones, } \\
\text { transiciones } \\
\text { diapositivas }\end{array}$ & $\begin{array}{l}\text { Ningún orden, excesivo o } \\
\text { ningún uso } \\
\text { animación y transición que } \\
\text { facilita distracción }\end{array}$ & $\begin{array}{l}\text { Orden lógico, aunque falta } \\
\text { animación y sólo aplica } \\
\text { transiciones en alguna } \\
\text { diapositiva, o efectúa ambas } \\
\text { de forma inadecuada }\end{array}$ & $\begin{array}{l}\text { Orden lógico, animación de } \\
\text { puntos no importantes o } \\
\text { transiciones que dificultan } \\
\text { atención }\end{array}$ & $\begin{array}{c}\text { Animación puntos } \\
\text { importantes, ayuda a centrar } \\
\text { la atención en lo importante y } \\
\text { evita distraerse }\end{array}$ \\
\hline
\end{tabular}

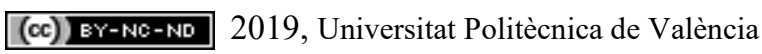




\begin{tabular}{|c|c|c|c|c|}
\hline \multicolumn{5}{|c|}{ 4. Habilidades de comunicación $(20 \%)$} \\
\hline & Inadecuado 0 & A mejorar 1 & Adecuado 2 & Muy adecuado 3 \\
\hline $\begin{array}{l}\text { Volumen y tono } \\
\text { de voz }\end{array}$ & $\begin{array}{l}\text { Demasiado bajo para ser } \\
\text { escuchado por todos y } \\
\text { excesivamente monótono }\end{array}$ & $\begin{array}{l}\text { Suficientemente alto para ser } \\
\text { escuchado alrededor del } 70 \% \text { del } \\
\text { tiempo por todos y } / \mathrm{o} \text { tono } \\
\text { inadecuado (monótono, aburrido) }\end{array}$ & $\begin{array}{l}\text { Suficientemente alto para ser } \\
\text { escuchado por todos y tono de voz } \\
\text { adecuado alrededor del } 90 \%\end{array}$ & $\begin{array}{l}\text { Suficientemente alto y con tono } \\
\text { de voz adecuado para ser } \\
\text { escuchado por toda la audiencia }\end{array}$ \\
\hline $\begin{array}{l}\text { Claridad en el } \\
\text { habla }\end{array}$ & $\begin{array}{l}\text { Poca claridad. A menudo } \\
\text { masculla y pronuncia mal } \\
\text { palabras }\end{array}$ & $\begin{array}{c}\text { Habla claro } 85-90 \% \text { del tiempo y } \\
\text { pronuncia mal numerosas } \\
\text { palabras }\end{array}$ & $\begin{array}{c}\text { Habla claro el } 90-95 \% \text { del tiempo, } \\
\text { aunque se equivoca al pronunciar } \\
\text { algunas palabras }\end{array}$ & $\begin{array}{l}\text { Habla claro la práctica totalidad } \\
\text { del tiempo. }\end{array}$ \\
\hline $\begin{array}{l}\text { Pausas y uso de } \\
\text { coletillas }\end{array}$ & $\begin{array}{l}\text { No usa pausas de manera } \\
\text { intencionada Abuso de } \\
\text { coletillas. Gran cantidad de } \\
\text { frases incompletas }\end{array}$ & $\begin{array}{l}\text { Número de pausas inadecuado - } \\
\text { por exceso o defecto-, introduce } \\
\text { algunas en momentos } \\
\text { inesperados o utiliza } \\
\text { frecuentemente coletillas (um...., } \\
\text { ehhh..., bien...etc). Utiliza } \\
\text { muchas frases incompletas }\end{array}$ & $\begin{array}{l}\text { Usa las pausas correctamente, } \\
\text { aunque introduce algunas de forma } \\
\text { inesperada/ inadecuada, utiliza en } \\
\text { diversas ocasiones coletillas } \\
\text { (um..., ehhh..., bien...etc.). Utiliza } \\
\text { frases incompletas en más del } 5 \% \\
\text { ocasiones } \\
\end{array}$ & $\begin{array}{c}\text { Usa las pausas correctamente y } \\
\text { al final de las oraciones. Utiliza } \\
\text { frases completas en más del } \\
95 \% \text { ocasiones }\end{array}$ \\
\hline $\begin{array}{c}\text { Postura y } \\
\text { contacto visual }\end{array}$ & $\begin{array}{l}\text { No mantiene contacto visual y la } \\
\text { postura corporal es inadecuada }\end{array}$ & $\begin{array}{l}\text { A veces mantiene postura } \\
\text { erguida y mantiene contacto } \\
\text { visual con la audiencia, pero } \\
\text { menos del } 90 \% \text { del tiempo }\end{array}$ & $\begin{array}{c}\text { Postura erguida y establece } \\
\text { contacto visual con todos, aunque } \\
\text { se observan algunos indicadores de } \\
\text { tensión/desinterés } \\
\end{array}$ & $\begin{array}{c}\text { Espalda recta, postura relajada y } \\
\text { confiada, establece contacto } \\
\text { visual con todos }\end{array}$ \\
\hline
\end{tabular}


Tabla 3. Apartado de apoyo visual de la rúbrica modificada (en rojo las modificaciones realizadas) para la exposición oral del grupo INTERVENCIÓN.

\begin{tabular}{|c|c|c|c|c|}
\hline \multicolumn{5}{|c|}{ 3. Apoyo visual ( $20 \%)$} \\
\hline & Inadecuado 0 & A mejorar 1 & Adecuado 2 & Muy adecuado 3 \\
\hline $\begin{array}{l}\text { Número y } \\
\text { explicación de las } \\
\text { diapositivas }\end{array}$ & $\begin{array}{c}\text { No cumple requisitos de número } \\
\text { (muchas ideas importantes en la } \\
\text { misma diapositiva) y adecuación } \\
\text { en su explicación. }\end{array}$ & $\begin{array}{c}\text { Utiliza un número inadecuado de } \\
\text { diapositivas (en ocasiones hay } \\
\text { diapositivas con muchas ideas } \\
\text { importantes en la misma } \\
\text { diapositiva), aunque intenta } \\
\text { explicarlas }\end{array}$ & $\begin{array}{l}\text { Cantidad de diapositivas adecuada } \\
\text { (como máximo una idea importante } \\
\text { por diapositiva), aunque imprecisa } \\
\text { explicación de alguna de ellas }\end{array}$ & $\begin{array}{c}\text { Cantidad de diapositivas } \\
\text { adecuada (como máximo una } \\
\text { idea importante por diapositiva) } \\
\text { y explicación pertinente de las } \\
\text { mismas }\end{array}$ \\
\hline $\begin{array}{c}\text { Adecuación de las } \\
\text { diapositivas }\end{array}$ & $\begin{array}{l}\text { Incorpora diapositivas no } \\
\text { pertinentes con la temática, no } \\
\text { facilitan la comprensión ni la } \\
\text { motivación de la audiencia. }\end{array}$ & $\begin{array}{l}\text { Aunque la mayoría de ellas están } \\
\text { relacionadas con el tema, no } \\
\text { favorecen su comprensión ni la } \\
\text { motivación de la audiencia }\end{array}$ & $\begin{array}{l}\text { Todas tiene relación con el tema y } \\
\text { facilitan que la comprensión } \\
\text { material, aunque algunas no están } \\
\text { conectadas con el discurso o } \\
\text { adolecen de amenidad }\end{array}$ & $\begin{array}{c}\text { Todas tiene relación con el tema } \\
\text { y conexión con el discurso, } \\
\text { facilitando la } \\
\text { comprensión del material y } \\
\text { resultan amenas/captan la } \\
\text { atención }\end{array}$ \\
\hline Legibilidad & $\begin{array}{c}\text { Ilegible, uso de colores } \\
\text { abrumador, abusa de texto y de } \\
\text { párrafos }\end{array}$ & $\begin{array}{l}\text { Letra legible por el } 85 \% \\
\text { audiencia aproximad., extension } \\
\text { excesiva en gran parte } \\
\text { diapositivas. Inadecuado } \\
\text { contraste de colores }\end{array}$ & $\begin{array}{l}\text { Letra legible para toda la audiencia, } \\
\text { en algunas ocasiones mucho texto } \\
\text { por diapositiva. Contraste adecuado } \\
\text { de colores }\end{array}$ & $\begin{array}{c}\text { Cantidad de texto adecuada por } \\
\text { diapositiva, letra legible para } \\
\text { toda la audiencia, contraste } \\
\text { adecuado de colores }\end{array}$ \\
\hline $\begin{array}{c}\text { Relevancia y } \\
\text { adecuación } \\
\text { imágenes/esquem } \\
\text { as }\end{array}$ & No utiliza o distraen & $\begin{array}{c}\text { Acorde con contenido, aunque } \\
\text { no relevantes y de poca } \\
\text { resolución }\end{array}$ & $\begin{array}{l}\text { Relevantes, acordes con contenidos } \\
\text { en la mayoría de ocasiones, pero } \\
\text { poca resolución }\end{array}$ & $\begin{array}{c}\text { Relevantes, acordes con } \\
\text { contenidos y con adecuada } \\
\text { resolución }\end{array}$ \\
\hline $\begin{array}{l}\text { Secuenciación, } \\
\text { animaciones, } \\
\text { transiciones } \\
\text { diapositivas }\end{array}$ & $\begin{array}{l}\text { Ningún orden, excesivo o } \\
\text { ningún uso } \\
\text { animación y transición que } \\
\text { facilita distracción }\end{array}$ & $\begin{array}{l}\text { Orden lógico, aunque falta } \\
\text { animación y sólo aplica } \\
\text { transiciones en alguna } \\
\text { diapositiva, o efectúa ambas de } \\
\text { forma inadecuada }\end{array}$ & $\begin{array}{l}\text { Orden lógico, animación de puntos } \\
\text { no importantes o transiciones que } \\
\text { dificultan atención }\end{array}$ & $\begin{array}{l}\text { Animación puntos importantes, } \\
\text { ayuda a centrar la atención en lo } \\
\text { importante y evita distraerse }\end{array}$ \\
\hline
\end{tabular}

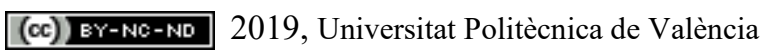




\subsection{Cuestionario de percepción de validez de la rúbrica}

Se desarrolló un cuestionario dirigido a evaluar la percepción de los estudiantes sobre la validez y utilidad de la rúbrica. Este cuestionario estuvo compuesto por 9 ítems (ver ítems en Tabla 4), utilizando una escala de respuesta tipo Likert de 5 niveles, desde "nada de acuerdo" hasta "muy de acuerdo". El cuestionario fue realizado por el alumnado de manera anónima, siendo 35 el total de participantes del grupo INTERVENCIÓN y 59 el del grupo CONTROL.

\subsection{Valoración del rendimiento académico}

Se utilizaron las calificaciones obtenidas por los distintos grupos de estudiantes en las exposiciones orales, tanto en los cuatro subapartados de la rúbrica (principios básicos, aplicación y ejemplificación, apoyo visual, y habilidades de comunicación), como en la valoración final, así como la calificación final de la asignatura. Dichas calificaciones se utilizaron como valores de control y discusión de ambos grupos.

\subsection{Análisis estadístico}

Para el análisis estadístico se utilizó el programa SPSS (SPSS Statistics v.21, IBM, Nueva York, EEUU). En primer lugar, en el estudio se han desarrollado distintos análisis complementarios dirigidos a evaluar la estructura factorial y consistencia interna de la escala de percepción de validez de la rúbrica, así como a determinar y a comparar la percepción de validez de la rúbrica en los dos grupos participantes en el estudio (INTERVENCION y CONTROL). Para determinar la estructura factorial de percepción de validez se efectuó un Análisis Factorial Exploratorio a través del método de ejes principales, determinando la consistencia interna de las dimensiones resultantes a través del alfa de Cronbach. Por otro lado, se obtuvieron los descriptivos básicos de las puntuaciones obtenidas por los estudiantes en las exposiciones orales a través de la aplicación de la rúbrica de evaluación, considerando tanto la puntuación total que ofrece la rúbrica como la puntuación obtenida en cada uno de sus subapartados (principios básicos, aplicación y ejemplificación, apoyo visual y habilidades de comunicación). Dado que las puntuaciones en los subapartados de principios básicos y de aplicación y ejemplificación, así como en nota final de la asignatura, mostraron una distribución no normal (test Kolgomorov-Smirnov, $p<0,05$ ) se utilizó en estos casos el test U de Mann-Whitney para evaluar las diferencias entre los dos grupos participantes (INTERVENCIÓN y CONTROL). En el resto de comparaciones entre grupos -calificación global de la exposición y puntuaciones en los subapartados de la rúbrica de apoyo visual y habilidades comunicativas- se aplicó una prueba T-Student. En las comparaciones se utilizó $\mathrm{p}<0,05$ como límite de significación estadística. 


\section{Resultados}

\subsection{Características psicométricas del cuestionario de percepción de la validez y utilidad de la rúbrica}

Un conjunto de análisis preliminares $\left(\mathrm{KMO}=0,74\right.$; Prueba de esfericidad de Bartlett, $X^{2}(36)$ $=191,0, \mathrm{p}<0,001)$ constató la adecuación de los datos para aplicar técnicas de análisis factorial exploratorio. Los resultados del mismo, desarrollado a través del método de ejes principales, evidenció la estructura unifactorial de la escala de percepción de validez y utilidad de la rúbrica (Eigenvalue = 2,4; Explica el 26,5\% de la varianza de las respuestas). Por otro lado, sus características psicométricas resultaron satisfactorias, considerando tanto su consistencia interna (alfa de Cronbach $=0,74$ ) como los índices de discriminación de sus ítems (correlación ítem total promedio de 0,42 ), permitiendo obtener una valoración total de la escala a partir de la suma de todos sus ítems.

\subsection{Efectos de la implicación en el desarrollo de la rúbrica en la percepción de la validez y utilidad de la rúbrica}

Los valores de la percepción de la validez de la rúbrica se muestran en la Tabla 4. En primer lugar, teniendo en cuenta los datos de la totalidad del alumnado, se puede observar como la rúbrica fue valorada muy positivamente obteniendo en la mayoría de los casos valores promedio superiores al 4 (correspondiente a las valoraciones entre "De acuerdo" y "Muy de acuerdo").

El grupo INTERVENCIÓN obtuvo una peor valoración total de la validez de la rúbrica respecto al CONTROL. De manera más específica, los dos ítems que fueron peor valorados por el grupo INTERVENCIÓN respecto al CONTROL fueron los relacionados sobre si era adecuado el número de ítems y si la rúbrica había servido para preparar la exposición. Estos resultados están en contra de lo que se podría esperar, ya que se imaginaba que las mejores valoraciones se darían en el grupo que se había podido implicar en la modificación de la rúbrica. En este sentido, existe poca literatura que haya analizado el efecto de la participación del alumnado en la creación de la rúbrica, destacando el estudio de Fraile, Panadero y Pardo (2017) en el que observaron que la implicación en la creación de la rúbrica mejoraba la autorregulación y autoeficacia hacia el aprendizaje y el rendimiento académico de los estudiantes. No obstante, es importante destacar que dicho grupo realizó pequeñas modificaciones en un escaso número de componentes de la rúbrica relacionados con el apoyo visual. Por tanto, posiblemente no acabaron percibiendo la rúbrica como más "propia" que el grupo control, ya que el resto de subapartados de la rúbrica no fueron modificados. 
Tabla 4. Media y desviación estándar (DE) de la valoración de la percepción de validez de la rúbrica de todo el alumnado, grupo CONTROL y grupo INTERVENCIÓN (INTERV.) y p valores de las diferencias entre ambos grupos.

\begin{tabular}{llccc}
\hline \multicolumn{1}{c}{ Ítem } & \multicolumn{1}{c}{ Media (DE) sobre un máximo de 5 puntos } & Dif \\
\hline \multicolumn{1}{c}{ TOTAL } & CONTROL & INTERV. & p \\
\hline $\begin{array}{l}\text { 1.Me ha parecido muy apropiada para valorar las } \\
\text { exposiciones. }\end{array}$ & $4,2(0,5)$ & $4,2(0,6)$ & $4,1(0,5)$ & 0,47 \\
\hline $\begin{array}{l}\text { 2.Me ha parecido muy fácil de utilizar. } \\
\text { 3.El número de ítems era adecuado. }\end{array}$ & $4,4(0,7)$ & $4,4(0,7)$ & $4,4(0,8)$ & 0,92 \\
\hline $\begin{array}{l}\text { 4.Era fácil en cada ítem saber la valoración de } \\
\text { cada grupo. }\end{array}$ & $3,6(0,8)$ & $3,7(0,8)$ & $3,5(0,8)$ & 0,30 \\
\hline $\begin{array}{l}\text { 5.Teniendo la rúbrica es más fácil preparar una } \\
\text { exposición para conseguir buena nota. }\end{array}$ & $4,6(0,6)$ & $4,6(0,7)$ & $4,5(0,6)$ & 0,36 \\
\hline $\begin{array}{l}\text { 6.Con la rúbrica se puede ser más justo en la } \\
\text { evaluación. }\end{array}$ & $4,2(0,7)$ & $4,2(0,6)$ & $4,2(0,8)$ & 0,80 \\
\hline $\begin{array}{l}\text { 7.La rúbrica nos ha ayudado a preparar la } \\
\text { exposición. }\end{array}$ & $4,3(0,9)$ & $4,4(0,7)$ & $3,9(1,0)$ \\
\hline $\begin{array}{l}\text { 8.La rúbrica recoge los aspectos más } \\
\text { importantes a evaluar de la exposición. }\end{array}$ & $4,2(0,7)$ & $4,3(0,7)$ & $4,1(0,7)$ & $\mathbf{0 , 0 0 7}$ \\
\hline $\begin{array}{l}\text { 9.Recomiendo que se siga utilizando la rúbrica } \\
\text { en futuros cursos. }\end{array}$ & $4,4(0,7)$ & $4,4(0,6)$ & $4,3(0,7)$ & 0,20 \\
\hline $\begin{array}{l}\text { 10.Valoración total (suma de todos los ítems; } \\
\text { valoración máxima=45). }\end{array}$ & $37,9(3,7)$ & $38,5(3,5)$ & $36,8(3,9)$ & 0,51 \\
\hline
\end{tabular}

\subsection{Valoración del rendimiento académico de ambos grupos}

Aunque no se observaron diferencias entre ambos grupos en los cuatro grandes apartados que integra la rúbrica analizados por separado ( $\mathrm{p}>0,05)$, como tendencia el grupo control mostró valores ligeramente superiores (Fig. 1). Sin embargo, el grupo CONTROL sí obtuvo mejores resultados globales que el grupo INTERVENCIÓN, al presentar una mejor calificación final de la exposición oral, así como en la evaluación final de la asignatura $(\mathrm{p}<0,05)$. Esto apunta a que el rendimiento académico previo (el grupo CONTROL corresponde al de idioma valenciano donde los estudiantes matriculados suelen mostrar niveles de rendimiento académico en la titulación superiores) puede que tenga más influencia tanto sobre la percepción de validez y utilidad de la rúbrica como sobre los resultados finales obtenidos tras su aplicación que el hecho de haber podido participar en su modificación. Así, a modo de ejemplo, el ítem 7 (Tabla 4) fue uno de los que estuvo peor valorado por el grupo INTERVENCIÓN en comparación con el grupo CONTROL. Dicho ítem trata sobre utilizar la rúbrica en la preparación de la exposición y puede estar mejor valorado por el alumnado más trabajador e implicado. La relación entre rendimiento académico individual y percepción de validez de la rúbrica no ha sido analizada en profundidad por la literatura existente, por lo que se recomiendan futuros estudios que investiguen este aspecto. 


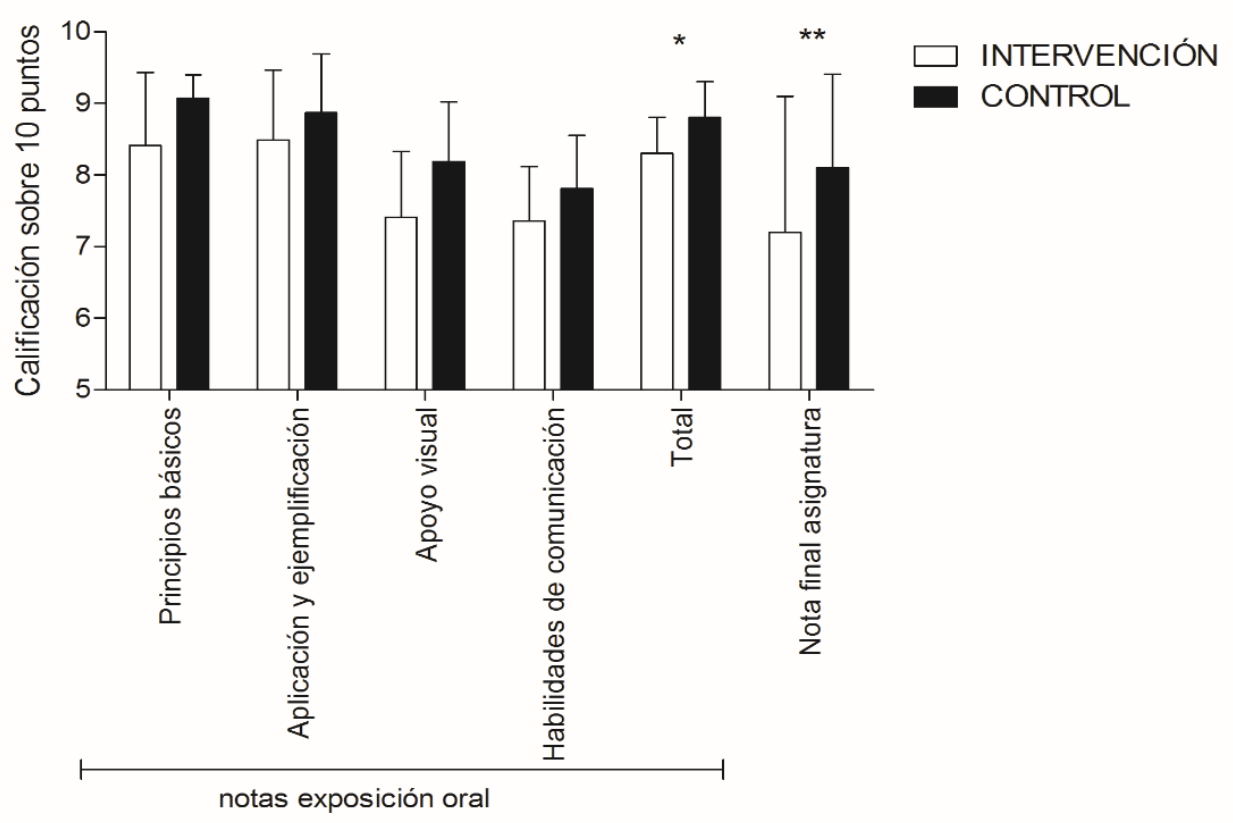

Fig. 1 Media y desviación estándar de las calificaciones obtenidas por el grupo INTERVENCIÓN y grupo CONTROL. Las diferencias se muestran mediante $*(p<0,05)$ y** $(p<0,01)$.

\section{Conclusiones}

El alumnado en su conjunto valoró positivamente la validez de la rúbrica como método de preparación y evaluación de la exposición oral. Los resultados no muestran efectos significativos de la implicación de los estudiantes en la construcción y modificación de la rúbrica sobre la percepción de la validez y utilidad de la misma. No obstante, hay que tener en cuenta que el alumnado que tuvo posibilidad de modificar la rúbrica, realizó pocas y pequeñas modificaciones, por lo que posiblemente su percepción sobre la rúbrica no se vio lo suficientemente alterada como para distinguirse del grupo control en este sentido. Sin embargo, el rendimiento académico sí que pudo tener una mayor influencia, por lo que resulta necesario controlar los efectos del rendimiento previo de los estudiantes en la titulación a la hora llevar a cabo una futura intervención.

\section{Referencias}

ANDRADE, H. y DU, Y., (2005). "Student Perspectives on Rubric-Referenced Assessment"en Educational \& Counseling Psychology Faculty Scholarship.

BARNEY, S., KHURUM, M., PETERSEN, K., UNTERKALMSTEINER, M., y JABANGWE, R., (2012). "Improving Students With Rubric-Based Self-Assessment and Oral Feedback" en IEEE Transactions on Education, vol. 55, issue 3, p. 319-325.

CROSLING, G. y WARD, I., (2002). "Oral communication: the workplace needs and uses of business graduate employees" en English for Specific Purposes, vol. 21, issue 1, p. 41-57. 
DASÍ, A. y IBORRA, M., (2007). "La evaluación de los estudiantes en la Educación Superior.La mejora de la comunicación oral: una perspectiva de proceso". Servei de Formació Permanent. Universitat de València.

DUNBAR, N.E., BROOKS, C.F., y KUBICKA-MILLER, T., (2006). "Oral Communication Skills in Higher Education: Using a Performance-Based Evaluation Rubric to Assess Communication Skills" en Innovative Higher Education, vol. 31, issue 2, p. 115.

ESHUN, E.F. y OSEI-POKU, P., (2013). "Design Students Perspectives on Assessment Rubric in Studio-Based Learning" en Journal of University Teaching and Learning Practice, vol. 10 , issue 1 .

FRAILE, J., PANADERO, E., y PARDO, R. (2017). "Co-creating rubrics: The effects on self-regulated learning, self-efficacy and performance of establishing assessment criteria with students" en Studies in Educational Evaluation, vol. 53, p. 69-76.

GARCÍA-ROS, R. (2011). "Análisis y validación de una rúbrica para evaluar habilidades de presentación oral en contextos universitarios" en Electronic Journal of Research in Educational Psychology, vol. 9, issue 25.

JONSSON, A. y SVINGBY, G., (2007). "The use of scoring rubrics: Reliability, validity and educational consequences" en Educational research review, vol. 2, issue 2, p. 130-144.

REDDY, Y.M. y ANDRADE, H., (2010). "A review of rubric use in higher education" en Assessment \& evaluation in higher education, vol. 35, isse 4, p. 435-448.

ŽIVKOVIĆ, S., (2014). "The Importance Of Oral Presentations For University Students" en Mediterranean Journal of Social Sciences, vol. 5, issue 19, p. 468. 